\title{
Association between weight change and incidence of cardiovascular disease events and mortality among adults with type 2 diabetes: a systematic review of observational studies and behavioural intervention trials
}

\author{
Jean Strelitz ${ }^{1}$ (D) Emma R. Lawlor ${ }^{1}$ (D) $\cdot$ Yue Wu $^{2} \cdot$ Annabel Estlin $^{1}$ - Giri Nandakumar ${ }^{2}$. Amy L. Ahern ${ }^{1}$ (D) \\ Simon J. Griffin ${ }^{1,3}$ (D)
}

Received: 26 May 2021 / Accepted: 25 August 2021 / Published online: 2 December 2021

(C) The Author(s) 2021

\begin{abstract}
Aims/hypothesis Weight loss is often recommended in the treatment of type 2 diabetes. While evidence has shown that large weight loss may lead to diabetes remission and improvement in cardiovascular risk factors, long-term impacts are unclear. We performed a systematic review of studies of weight loss and other weight changes and incidence of CVD among people with type 2 diabetes. Methods Observational studies of behavioural (non-surgical and non-pharmaceutical) weight changes and CVD events among adults with type 2 diabetes, and trials of behavioural interventions targeting weight loss, were identified through searches of MEDLINE, EMBASE, Web of Science, CINAHL, and The Cochrane Library (CENTRAL) until 9 July 2019. Included studies reported change in weight and CVD and/or mortality outcomes among adults with type 2 diabetes. We performed a narrative synthesis of observational studies and meta-analysis of trial data.

Results Of 13,227 identified articles, 17 (14 observational studies, three trials) met inclusion criteria. Weight gain (vs no change) was associated with higher hazard of CVD events (HRs [95\% CIs] ranged from 1.13 [1.00, 1.29] to 1.63 [1.11, 2.39]) and all-cause mortality (HRs [95\% CIs] ranged from $1.26[1.12,1.41]$ to $1.57[1.33,1.85]$ ). Unintentional weight loss (vs no change) was associated with higher risks of all-cause mortality, but associations with intentional weight loss were unclear. Behavioural interventions targeting weight loss showed no effect on CVD events (pooled HR [95\% CI] 0.95 [0.71, 1.27]; $I^{2}=50.1 \%$ ). Risk of bias was moderate in most studies and was high in three studies, due to potential uncontrolled confounding and method of weight assessment. Conclusions/interpretation Weight gain is associated with increased risks of CVD and mortality, although there is a lack of data supporting behavioural weight-loss interventions for CVD prevention among adults with type 2 diabetes. Long-term follow-up of behavioural intervention studies is needed to understand effects on CVD and mortality and to inform policy concerning weight management advice and support for people with diabetes.
\end{abstract}

PROSPERO registration CRD42019127304.

Keywords Cardiovascular complications · Cardiovascular disease $\cdot$ Epidemiology $\cdot$ Meta-analysis $\cdot$ Systematic review $\cdot$ Type 2 diabetes $\cdot$ Weight change $\cdot$ Weight loss $\cdot$ Weight management

Jean Strelitz

jean.strelitz@mrc-epid.cam.ac.uk

1 MRC Epidemiology Unit, Institute of Metabolic Science, School of Clinical Medicine, University of Cambridge, Cambridge, UK

2 School of Clinical Medicine, University of Cambridge, Cambridge, UK

3 Primary Care Unit, Department of Public Health and Primary Care, School of Clinical Medicine, University of Cambridge, Cambridge, UK

\author{
Abbreviations \\ ACCORD Action to Control Cardiovascular \\ Risk in Diabetes \\ $\mathrm{CHF} \quad$ Congestive heart failure \\ MI Myocardial infarction \\ ROBINS-I Risk of Bias in Non-randomised \\ Studies of Interventions
}




\section{Research in context}

\section{What is already known about this subject?}

- Being overweight or obese is a strong risk factor for type 2 diabetes and CVD

- Data on the effects of weight loss and other weight changes on long-term outcomes, including CVD and mortality among people with diabetes, are conflicting

\section{What is the key question?}

- What evidence exists regarding the effect of weight change on risk of CVD among people with type 2 diabetes?

\section{What are the new findings?}

- Weight gain was consistently associated with higher risk of CVD events, although the impact of non-surgical weight loss on CVD remains unclear

- Pooling data from three randomised trials showed no evidence of an effect of behavioural interventions targeting weight loss on CVD endpoints

- Duration of weight loss, the method of accounting for unintentional weight loss, and the underlying CVD risk among participants contribute to heterogeneity in study findings

\section{How might this impact on clinical practice in the foreseeable future?}

- There is little direct evidence for the effect of weight loss on CVD risk among adults with type 2 diabetes; long-term follow-up of behavioural intervention studies is needed to inform the treatment of type 2 diabetes and the use of healthcare resources for weight management support in diabetes care

\section{Introduction}

Being overweight or obese is a strong risk factor for type 2 diabetes and CVD. International guidelines recommend that people with type 2 diabetes who are obese or overweight should be encouraged to make behavioural changes to achieve sustained weight loss. Weight loss among overweight or obese people may lead to diabetes remission [1] and improvements in some cardiovascular risk factors [2], although the impact on risk of CVD events remains unclear. Large weight loss following bariatric surgery is associated with a reduced risk of mortality and CVD among people with type 2 diabetes [3]. However, most individuals with type 2 diabetes do not receive bariatric surgery but are typically given weight management advice including education on healthy behaviour change. Such advice may result in weight maintenance or other changes in weight. However, the long-term effects of advice and changes in weight on CVD and mortality remain uncertain. Glucose-lowering medications that cause weight loss have shown cardiovascular benefits in some instances; however, these benefits may occur through other pleiotropic pathways $[4,5]$. As weight management advice and support are integral to diabetes prevention and treatment, the impact on long-term health should be considered. In order to synthesise the existing evidence, we performed a systematic review of research on the association between weight changes and risk of CVD events and mortality among adults with type 2 diabetes. We aimed to summarise evidence regarding the impact of weight loss achieved through behaviour modification on the incidence of CVD and premature mortality, which may inform advice to practitioners and patients about weight management after diabetes diagnosis.

\section{Methods}

Protocol registration The protocol was registered on PROSPERO (CRD42019127304) prior to article screening, based on the PRISMA statement [6].

Study eligibility Studies eligible for inclusion recruited adults (aged $\geq 18$ years) with a diagnosis of type 2 diabetes. We included observational studies that reported associations between change in weight/BMI (or other metrics of body composition) and CVD events and/or all-cause mortality. Weight data had to be collected at least twice to ascertain changes. Studies reported on CVD events (non-fatal CVD events, CVD mortality, myocardial infarction [MI], stroke, congestive heart failure [CHF]) and/or all-cause or CVDrelated mortality. No exclusion criteria were applied related to study design. We did not include studies of weight changes following bariatric surgery as bariatric surgery may affect CVD risk through mechanisms other than weight loss (e.g. alterations to the gut microbiome [7-9]). Similarly, trials of medications that influence weight were excluded as such medications may have pleiotropic effects on CVD risk. 
Studies including participants without diabetes were included if the results were presented separately for the participants with diabetes.

We included trials of the effects of behavioural interventions on CVD events among adults with type 2 diabetes in which the primary outcome included CVD events and/or mortality. The intervention needed to target physical activity and/or diet for the purpose of weight loss. No restrictions were placed on intervention duration, delivery mode or trial design. Eligible comparisons were as follows: (1) no intervention/ minimal intervention; (2) standard care; and (3) other behavioural programmes.

Studies needed to report cardiovascular events including MI, stroke and other CVD-related death. Incident CVD events could occur any time after the intervention period or after the time period in which weight change was assessed. Only published peer-reviewed research articles in the English language were included.

Search strategy and selection criteria Searches of the electronic databases MEDLINE, EMBASE, Web of Science, CINAHL and The Cochrane Library (CENTRAL) from inception to 9 July 2019 were conducted by EL and JS with input from a medical librarian. Searches included the following keywords and subject headings: (1) diabetes AND; (2) CVD events AND; (3) weight AND; and (4) study design. Searches were based on the MEDLINE search strategy (electronic supplementary material [ESM] Table 1), modified to include databasespecific terms. Terms for 'study design' were based on the SIGN search filters [10]. We searched reference lists of eligible studies and review articles to identify additional articles.

We imported search results into Covidence review management software [11]. Two reviewers (of JS, EL, YW, $\mathrm{AE}$ and GN) independently screened all abstracts. Full texts were screened for articles deemed to have potential eligibility or where eligibility was unclear. A third reviewer adjudicated if there was uncertainty or disagreement. For both screening stages, all researchers independently piloted an identical $10 \%$ of articles.

Data extraction Data on participant characteristics, weight assessment, interventions, analysis methods and results were independently extracted by JS and a second reviewer (EL, YW, AE or GN) using a template adapted from the Cochrane data extraction form [12], the Consolidated Standards of Reporting Trials 2010 statement [13] and the Template for Intervention Description and Replication checklist [14]. If details of the methods or study design were not available in the articles, we extracted information from related publications from the same study.

Risk of bias assessment Two researchers (JS, EL, YW, AE or GN) assessed studies independently using the Risk of Bias 2.0 tool or a modified version of the Risk of Bias in Nonrandomised Studies of Interventions (ROBINS-I) tool, depending on study design. We adapted the ROBINS-I tool to suit observational studies and considered bias related to the following factors: confounding; selection of participants; weight assessment; diabetes assessment; missing data; measurement of outcomes; and study design. Based on these criteria, studies were determined as being at 'low', 'moderate', 'serious' or 'critical' risk of bias, or were designated as 'no information'. The tools were piloted by all researchers for three studies $(15 \%)$ to ensure consistency. Results are presented using robvis [15].

Synthesis of results We meta-analysed the HRs from the intervention studies using a DerSimonian and Laird [16] random effects model and summarised heterogeneity between the studies using the $I^{2}$ statistic. We did not meta-analyse the observational studies due to the heterogeneity of study designs and outcomes and instead conducted a narrative synthesis. Forest plots were generated using Stata (Version 16.1; StataCorp, College Station, TX, USA).

\section{Results}

We screened 13,227 titles and abstracts and assessed 257 full text articles. Four additional articles [17-20] were identified from review reference lists. In total, 17 articles [17-33] met our inclusion criteria (Fig. 1). The main reasons for the exclusion of articles were that they did not contain original research or did not report on CVD events or mortality.

Study characteristics Fourteen [17-30] of the studies were observational studies, and three [31-33] were RCTs. One study [22] consisted of a post hoc analysis pooling primary data from three clinical trials [34-36]. Studies were conducted in Europe [21-23, 25, 28, 30, 31], the USA [17-20, 22, 24, 26, 29, 33], Japan [32] and South Korea [27], and one study was multinational [22] (Table 1).

Participant characteristics Sample sizes ranged from 444 [28] to 173,246 [27]. Baseline mean age ranged from 46 to 64 years. Diabetes duration was $\leq 1$ year in five studies [ 21 , $23,27,28,30],>1$ year in six studies [18, 19, 22, 24-26], and was not specified in three studies [17, 20, 29]. In most studies, diabetes status was derived from medical records, aside from two studies $[20,30]$ that included screening for type 2 diabetes at enrolment and three studies $[17,19,24]$ that relied on participant report of diabetes diagnosis.

Seven studies [22-24, 27, 28, 31, 32] excluded individuals with a history of CVD, whereas inclusion criteria in two studies specified high CVD risk [18, 25]. Other studies excluded individuals with $\mathrm{BMI}<25 \mathrm{~kg} / \mathrm{m}^{2}$ [21, 26, 33], history of type 1 
Fig. 1 PRISMA diagram
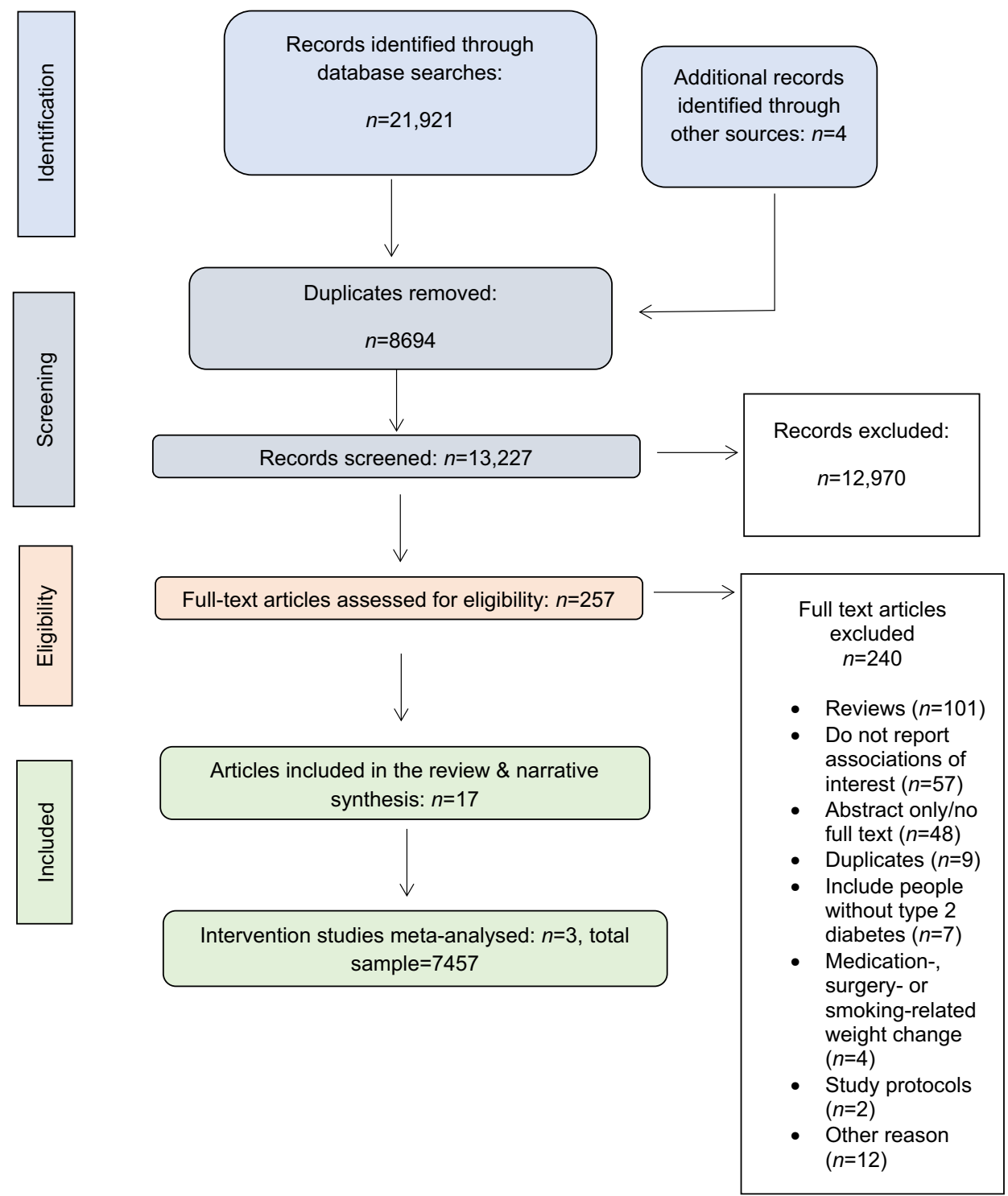

diabetes [25, 29] and history of cancer diagnosis [21, 24, 28, 31] (ESM Table 2). One study included women only [24].

\section{Characteristics of behavioural interventions targeting weight} loss All three RCTs [31-33] evaluated behavioural interventions with diet and physical activity components. One threearmed study [31] also included a pharmacological component; however, one treatment group only received the behavioural intervention, which we compared with the control arm. The intervention durations ranged from 4 years [33] to 8 years [32], delivered via face-to-face individual- and group-based sessions and one also included telephone counselling [32]. The comparator groups received conventional diabetes treatment $[31,32]$, and the comparator in one study also received education-based weight-loss support delivered in group sessions [33]. Hanefeld et al [31] and Sone et al [32] reported no changes in weight following the intervention. Wing et al
[33] reported greater weight loss in the intervention vs control group ( $8.6 \%$ vs $0.7 \%$ at 1 year).

Ascertainment and classification of weight changes in observational studies Weight measurements were ascertained from medical records in five observational studies [21, 23, 27-29]. Five studies included objective weight measurement $[18,20,25,26,30]$, and in three studies participants reported their weight [17, 19, 24]. One study provided no information on how weight was ascertained [22]. Most of the observational studies assessed weight change over a period of 1-2 years [19, 21, 23, 25-30]. For studies in which weight change was assessed over $\geq 2$ years, the time between weight measurements varied from 2 to 9 years $[17,24]$ and was not stated in one study [24]. In four studies, the duration of weight change varied [17, 18, 20, 22] (Table 1). 


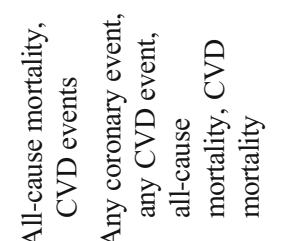

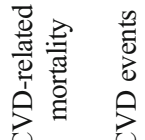

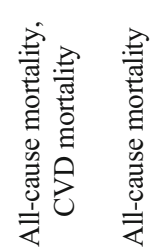

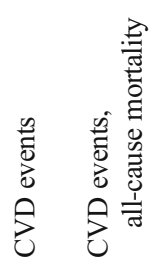

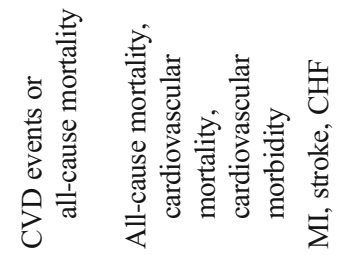

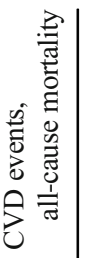

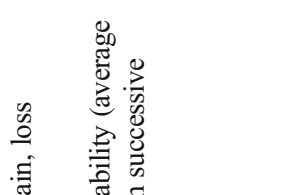

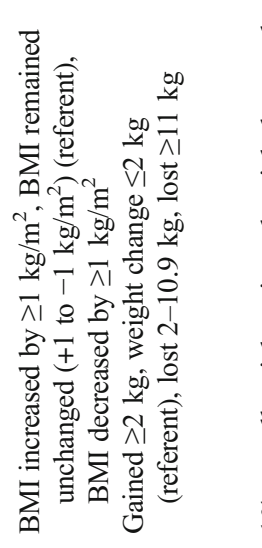

焉 尊

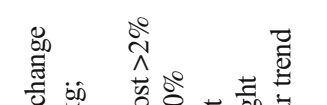

苟

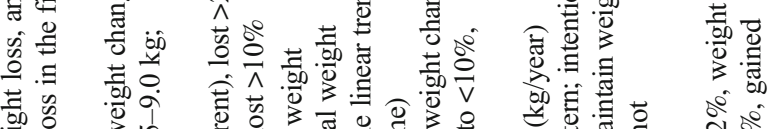

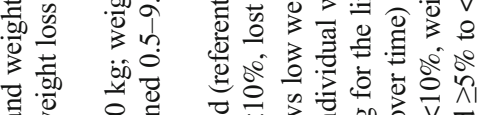

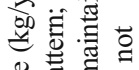

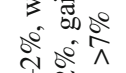

密 :

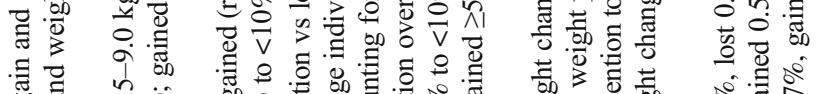

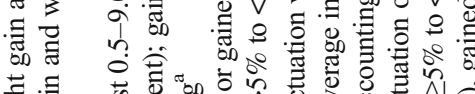

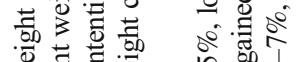

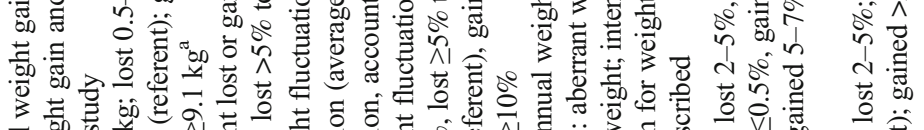

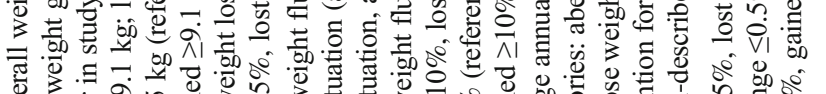

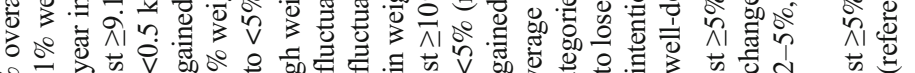

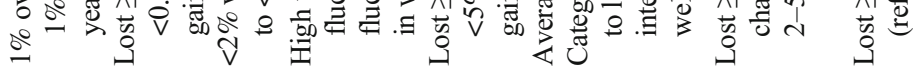

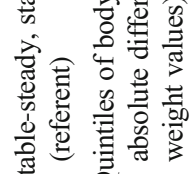

岩 总星

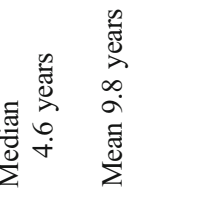

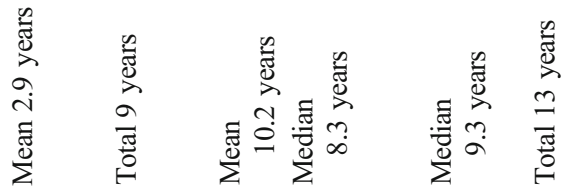

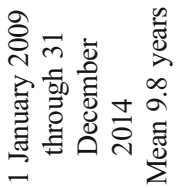

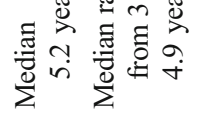

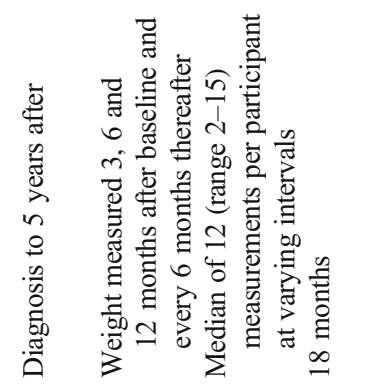

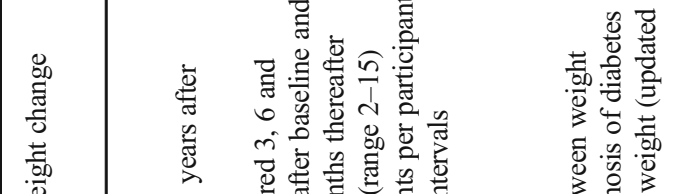

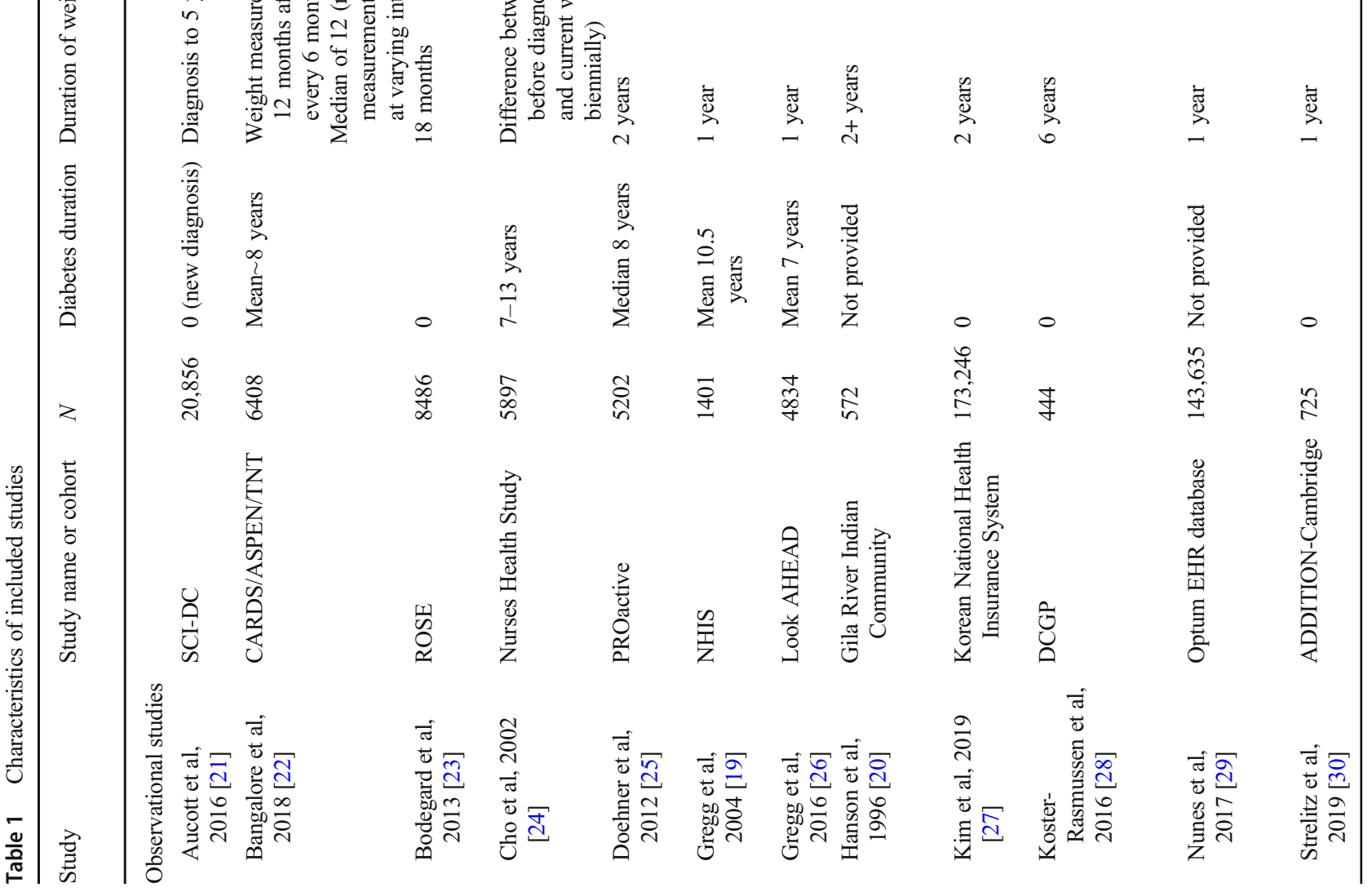




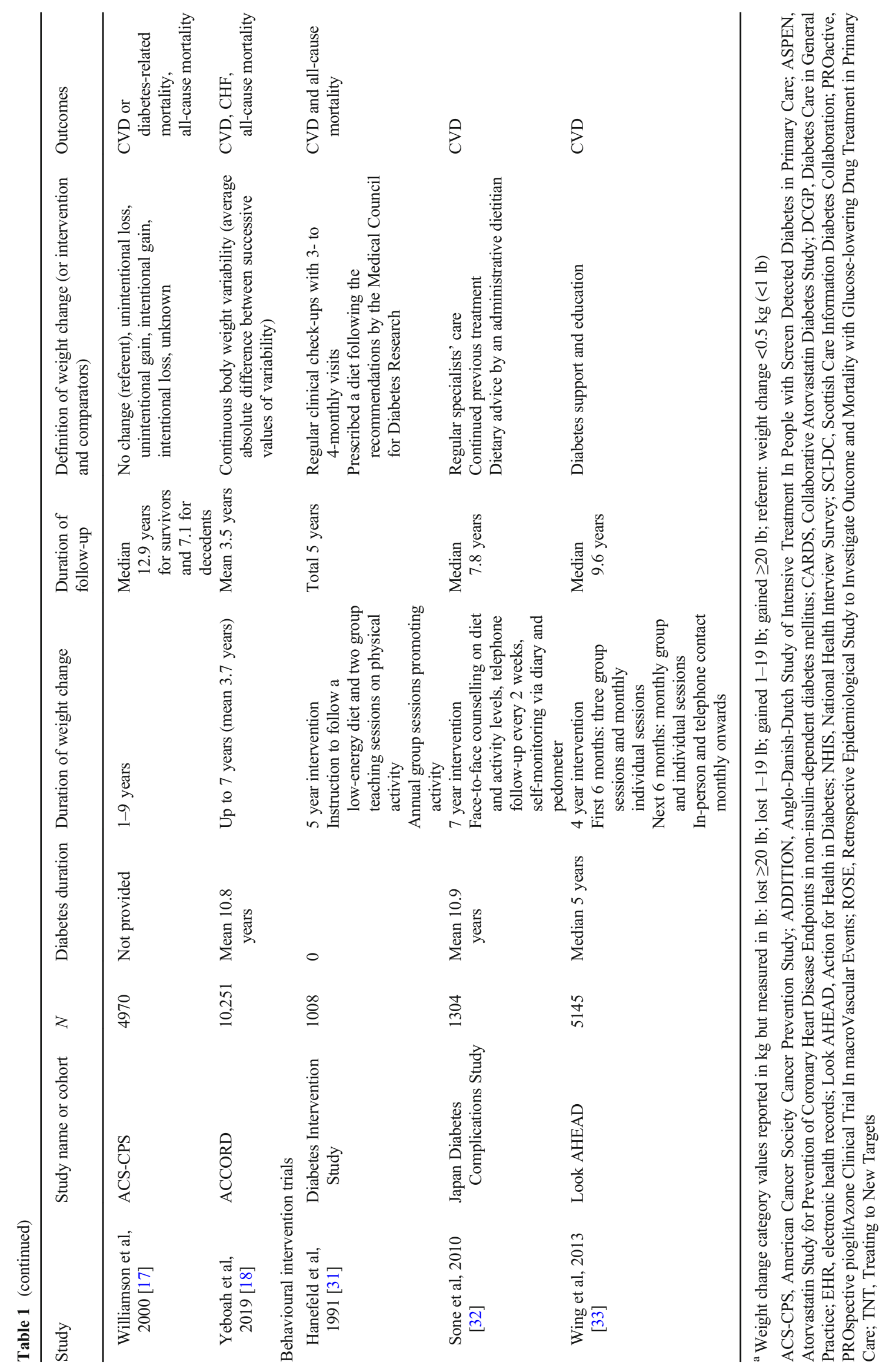


Six studies reported absolute weight change (kg or lb) [17, $18,22,24,28,29]$, one study reported change in BMI units $\left(\mathrm{kg} / \mathrm{m}^{2}\right)$ [23], five studies reported percentage weight change [25-27, 29, 30], and four studies focused on weight variability $[18,20-22]$. Most studies $(n=9)$ categorised changes in weight to distinguish weight gain, weight maintenance and weight loss, although definitions of the categories varied $[18,19,23-27,29,30]$. Three studies [17, 19, 28] assessed participants' self-reported intention to lose weight.

Assessment of cardiovascular events and mortality Most studies $(n=16)$ reported CVD outcomes, and many $(n=11)$ also reported all-cause mortality. In most studies, events were ascertained from medical records and/or mortality registries [17-24, 26-33]. One study did not provide details on the methods of outcome ascertainment [25]. Several studies [19, 21, 26-28, 30] included lags of 1-2 years between the assessment of weight changes and the risk period for mortality outcomes. The median or mean duration of follow-up for outcome events ranged from 2.9 to 10 years.

Risk of bias For the observational studies, risk of bias was rated to be moderate for all studies apart from three that were rated high risk of bias [17, 19, 24]. The main sources of bias were uncontrolled confounding, method of weight assessment, and method of diabetes classification (ESM Fig. 1). Of the three intervention studies, one was rated to have low risk of bias [32] and two moderate risk of bias [31, 33].

Associations between weight change, CVD and mortality in observational studies Weight gain was associated with an increased risk of CVD events [23, 27, 29]. Bodegard et al [23] reported that $\geq 1 \mathrm{~kg} / \mathrm{m}^{2}$ BMI gain after diabetes diagnosis was associated with higher CVD mortality compared with no BMI change (HR 1.63 [95\% CI 1.11, 2.39]) (Fig. 2). Kim et al [27] reported that a $\geq 10 \%$ weight gain after diabetes diagnosis was associated with a higher hazard of stroke (HR 1.47 [95\% CI $1.20,1.79]$ ) and all-cause mortality (HR 1.57 [95\% CI $1.33,1.85]$ ) but not MI events (HR 1.07 [95\% CI 0.84, 1.38]) (Fig. 3) [27]. Nunes et al [29] reported that weight gain $>7 \%$ was associated with higher hazard of MI (HR 1.13 [95\% CI 1.00, 1.29]) (Fig. 2) and reported similar results for stroke and CHF [29]. Strelitz et al [30] reported no association between weight gain and CVD events [30] (Fig. 2). Gregg et al [19] reported a higher hazard of all-cause mortality associated with weight gain $\geq 9.1 \mathrm{~kg}(\geq 20 \mathrm{lb}$ ), though the CI overlapped the null (HR 1.77 [95\% CI 0.97, 3.23]) (Fig. 2). The duration of follow-up in these studies ranged from 4.6 years to 9.8 years.

Two studies of weight change over $<2$ years reported protective associations between weight loss and CVD events. Gregg et al [26] and Strelitz et al [30] reported that $\geq 10 \%$ weight loss (vs weight gain or $<2 \%$ weight loss) and $\geq 5 \%$ weight loss (vs weight change $<2.5 \%$ ), respectively, were associated with a lower hazard of CVD over a mean followup of 10.2 years and 9.8 years. In contrast, Nunes et al [29] reported a higher hazard of MI following weight loss $\geq 5 \%$ (vs $<5 \%$ change) over 5 years, and Gregg et al [19] reported higher 9 year hazard of all-cause mortality for weight loss $\geq 20 \mathrm{lb}(\geq 9.1 \mathrm{~kg})$ vs no change. Bodegard et al [23] reported no association between a $\geq 1 \mathrm{~kg} / \mathrm{m}^{2}$ decrease in BMI over 18 months and risk of CVD mortality over a median of 4.6 years of follow-up (Fig. 2).

Studies of weight change over $2+$ years (Figs. 3 and 4) showed that weight loss and weight variability were associated with CVD events [22] and all-cause mortality [22, 27], particularly among individuals with history of CVD $[18,25]$ (Fig. 5). Bangalore et al [22] reported small but apparent associations between a $1 \mathrm{SD}$ increase in body weight variability and CVD morbidity and mortality (HR 1.08 [95\% CI 1.03, 1.14]) and all-cause mortality (HR 1.16 [95\% CI 1.10, 1.22]) over a median 5 years follow-up (Fig. 4) Yeboah et al [18] reported a higher hazard of CHF and CVD per 1 SD difference in body weight variability (HR 1.59 [95\% CI 1.45, 1.75] and 1.25 [95\% CI 1.15, 1.36], respectively) over a mean 3.5 years of follow-up among individual at high risk of CVD (Fig. 5). Other studies reported null associations between weight loss or weight variability over $\geq 2$ years and CVD events and allcause mortality [20, 21, 27, 28].

Six studies $[19,21,26-28,30]$ included a $1-2$ year lag in the risk period for all-cause mortality as a method for potentially attenuating the confounding effects of unintentional weight loss. In general, studies that included a lag showed null associations between weight change and mortality [19, 26, 28, 30], aside from one study by Kim et al [27] that reported both weight gain and loss (vs weight maintenance) were associated with higher all-cause mortality, with an apparent doseresponse relationship between weight change (both gain and loss) and mortality (Fig. 3).

Intentional and unintentional weight change Three studies assessed the impact of intentional vs unintentional weight changes on CVD and mortality outcomes by categorising participants based on their intention to lose weight [17, 19], or intention to lose or maintain weight [28]. Gregg et al [19] reported that weight loss among participants without intention to lose weight was associated with a higher hazard of all-cause mortality (HR 1.73 [95\% CI 1.20, 2.48]), though this association was attenuated after introducing a 2-year lag in the risk period for the outcome (Fig. 6). In the same study, participants who reported that they were trying to lose weight, but did not actually lose weight, had a lower hazard of all-cause mortality compared with participants who were not trying to lose weight (HR 0.74 [95\% CI 0.57, 0.98]). This study was determined to have high risk of bias related to confounding and self-reported assessment of weight and diabetes status (ESM Fig. 1). Koster- 
Study

Bodegard et al [23] - All-cause mortality

BMl loss $\geq 1 \mathrm{~kg} / \mathrm{m}^{2}$

Change $<1 \mathrm{~kg} / \mathrm{m}^{2}$ (ref)

BMl gain $\geq 1 \mathrm{~kg} / \mathrm{m}^{2}$

Bodegard et al [23] - CVD mortality

BMl loss $\geq 1 \mathrm{~kg} / \mathrm{m}^{2}$

Change $<1 \mathrm{~kg} / \mathrm{m}^{2}$ (ref)

BMI gain $\geq 1 \mathrm{~kg} / \mathrm{m}^{2}$

Gregg et al (2004) [19] - All-cause mortality

Any weight loss $(\mathrm{kg})$

Weight loss $\geq 9.1 \mathrm{~kg}$

Weight loss $0.5-9.0 \mathrm{~kg}$

No weight change (ref)

Any weight gain $(\mathrm{kg})$

Weight gain $0.5-9.0 \mathrm{~kg}$

Weight gain $\geq 9.1 \mathrm{~kg}$

Gregg et al (2016) [26] - CVD morbidity and mortality (1 year lag) $\geq 10 \%$ weight loss

$\geq 5 \%$ to $<10 \%$

$\geq 2 \%$ to $<5 \%$ weight loss

Gained or lost $<2 \%$ weight (ref)

Nunes et al [29] - Ml

Loss $\geq 5 \%$

Loss $\geq 2$ to $<5 \%$

Loss $\geq 0.5$ to $<2 \%$

$<5 \%$ weight loss or $\leq 5 \%$ weight gain (ref)

Gain $>0.5$ to $\leq 2.0 \%$

Gain $>2.0$ to $\leq 5.0 \%$

Gain $>5.0$ to $\leq 7.0 \%$

Gain $>7.0 \%$

Strelitz et al [30] - All-cause mortality (1 year lag)

$\geq 5 \%$ weight loss

$2-5 \%$ weight loss

$<2 \%$ weight change (ref)

Gained $>2 \%$ weight

Strelitz et al [30] - CVD morbidity and mortality

$\geq 5 \%$ weight loss

$2-5 \%$ weight loss

$<2 \%$ weight change (ref)

Gained $>2 \%$ weight
$\mathrm{HR}$

with $95 \% \mathrm{Cl}$

$1.06(0.85,1.32)$

$1.00(1.00,1.00)$

$1.34(1.01,1.76)$

$1.06(0.76,1.48)$

$1.00(1.00,1.00)$

$1.63(1.11,2.39)$

$1.22(0.99,1.50)$

$1.40(1.05,1.87)$

$1.11(0.87,1.41)$

$1.00(1.00,1.00)$

$1.11(0.74,1.66)$

$0.97(0.62,1.53)$

$1.77(0.97,3.23)$

$0.79(0.64,0.98)$

$1.16(0.95,1.42)$

$1.08(0.88,1.33)$

口 $1.00(1.00,1.00)$

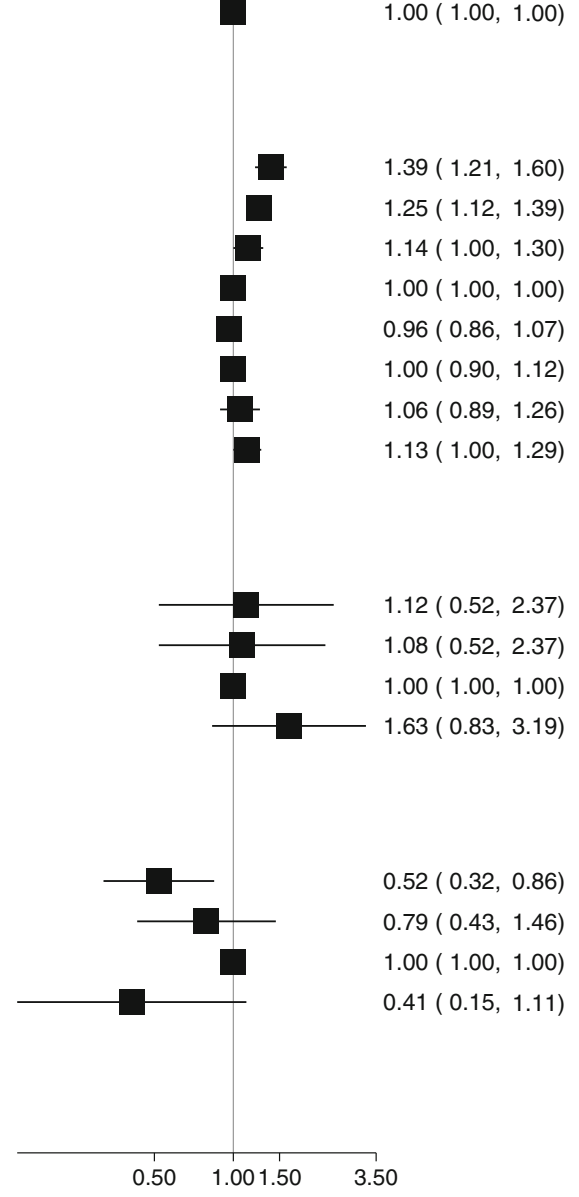


Fig. 2 Forest plot of HRs and 95\% CIs for the indicated outcome from studies of weight change over 1-2 years. Gregg et al [19] weight change category values reported in $\mathrm{kg}$ but measured in $\mathrm{lb}$ : lost $\geq 20 \mathrm{lb}$; lost $1-$ $19 \mathrm{lb}$; gained 1-19 lb; gained $\geq 20 \mathrm{lb}$; referent: weight change $<0.5 \mathrm{~kg}$ $(<1 \mathrm{lb})$. Ref, referent group

Rasmussen et al [28] reported no differences in the association between intentional vs unintentional weight loss and all-cause mortality, CVD morbidity or CVD mortality (Fig. 6).

Gregg et al [19] reported no association between intentional weight loss and all-cause mortality (HR 0.85 [95\% CI 0.66, 1.11]). Williamson et al [17] reported that intentional weight loss was associated with a lower hazard of death due to CVD or diabetes (HR 0.72 [95\% CI 0.63, 0.82]) and all-cause mortality (HR 0.75 [95\% CI 0.67, 0.84]). Both studies were determined to have a high risk of bias related to confounding, assessment of weight, and missing data (ESM Fig. 1).

Population subgroups: high CVD risk and women only In a study among participants with cardiovascular comorbidities, Doehner et al [25] reported a small increased risk of CVD mortality associated with $1 \%$ weight loss over 2 years (HR 1.07 [95\% CI 1.03, 1.10]) and increasingly stronger relationships between weight loss $\geq 5 \%, \geq 10 \%$ and $\geq 15 \%$ and allcause mortality (Fig. 5). Yeboah et al. reported that among the Action to Control Cardiovascular Risk in Diabetes
Fig. 3 Forest plot of HRs and 95\% CIs for the indicated outcome from studies in which the weight change interval was $\geq 2$ years. Ref, referent group

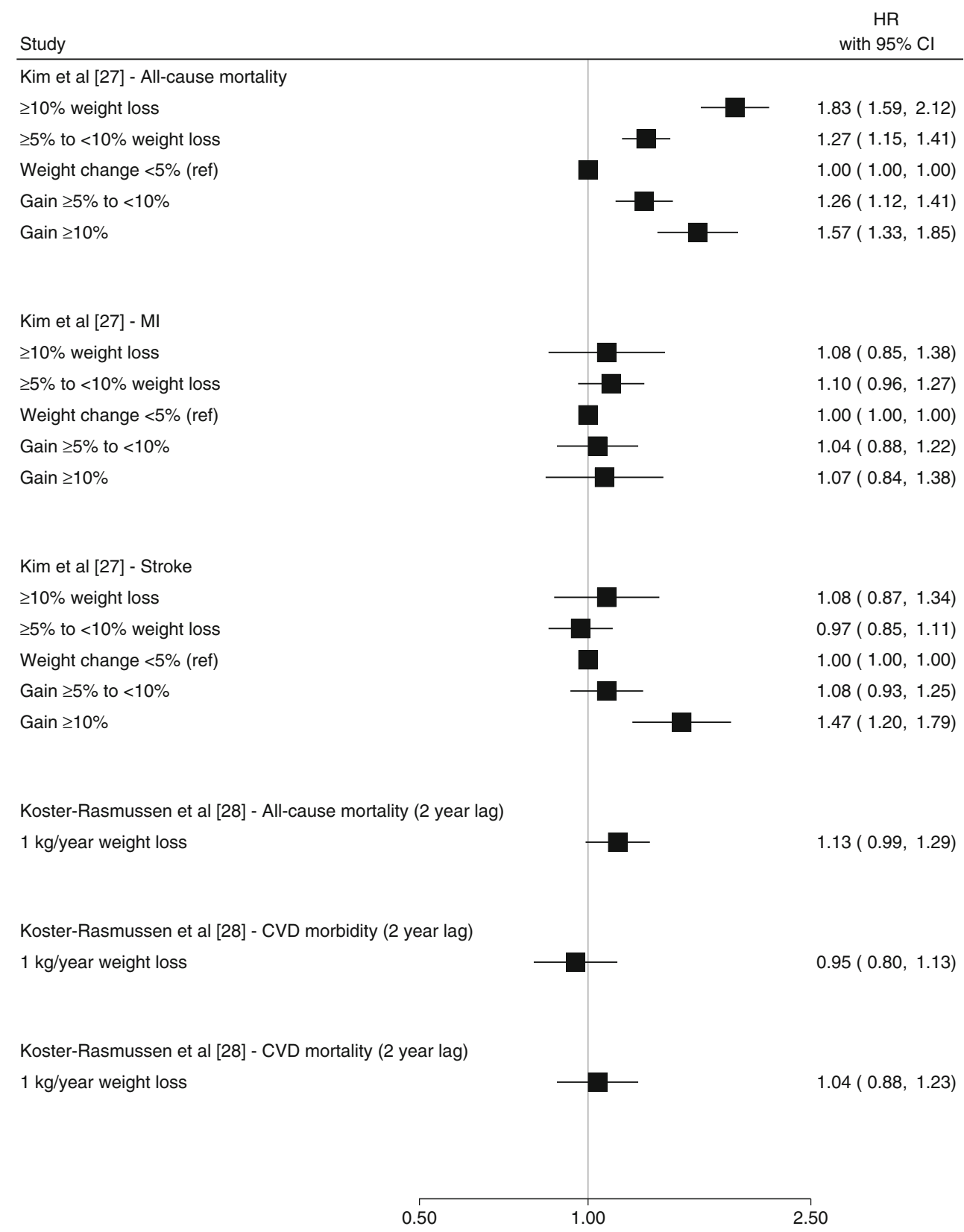


Fig. 4 Forest plot of HRs and 95\% CIs for the indicated outcome from studies of weight variability over $\geq 2$ years. Ref, referent group

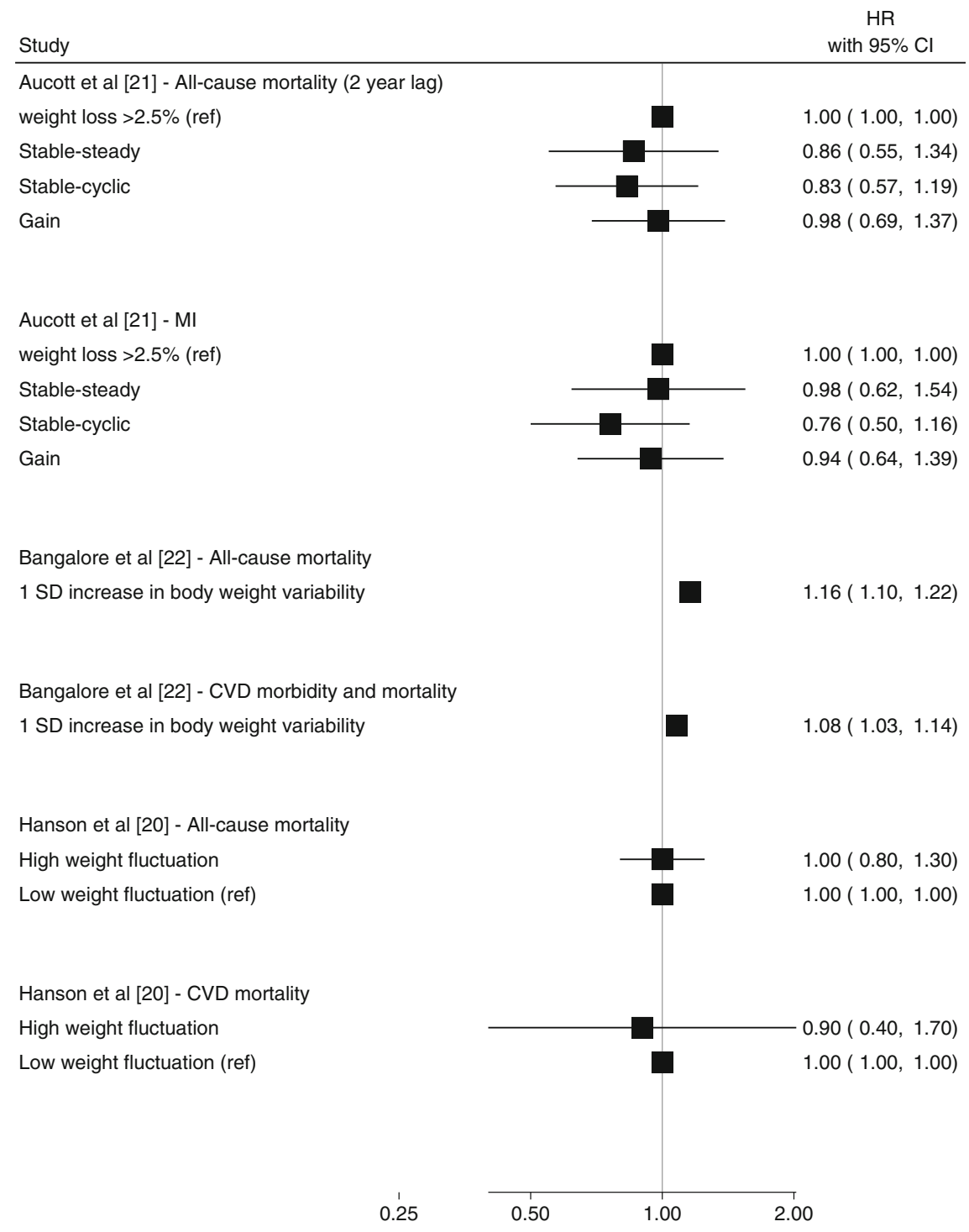

(ACCORD) study cohort of individuals with type 2 diabetes and high CVD risk, higher 7-year body weight variability was associated with higher hazard of CVD morbidity and mortality over a mean 3.5 years follow-up [18]. Among women enrolled in the Nurse's Health Study, Cho et al [24] reported no associations between weight gain or loss, where the duration of weight change was undefined, and risk of CVD over approximately 10 years of follow-up (Fig. 5).

\section{Effect of interventions targeting weight loss on CVD and} mortality Meta-analysis showed that behavioural interventions targeting weight loss had no effect on CVD outcomes (HR 0.95 [95\% CI 0.71, 1.27]), and heterogeneity was moderately high between studies $\left(I^{2}=50.1 \%\right)$ (Fig. 7). Of the three studies identified [31-33], one behavioural intervention reduced hazard of stroke by $38 \%$ (HR 0.62 [95\% CI 0.39 , 0.98]) over a median 7.8 years follow-up [32]. Wing et al found no effect on CVD incidence (HR 0.95 [95\% CI 0.83,
1.09]) over a median of 9.6 years follow-up [33]. Hanefeld et al reported that the MI incidence rate was higher in the intervention group (53.6 per 1000) vs the control group (30.3 per 1000) (rate ratio 1.77 [95\% CI 0.81, 3.87]) over a total of 5 years, and found no effect of the intervention on ischaemic heart disease risk (1.08 [95\% CI 0.65, 1.79]) [31] (Fig. 7). CIs for the rate ratio were manually calculated based on the numbers of events, as SEs were not provided in the publication.

\section{Discussion}

Behavioural interventions targeting weight loss among people with type 2 diabetes had no effect on 5-10 year risk of CVD outcomes. Synthesis of observational studies showed that weight gain after diabetes diagnosis was associated with higher hazards of stroke [27], CVD mortality [23, 28] and 


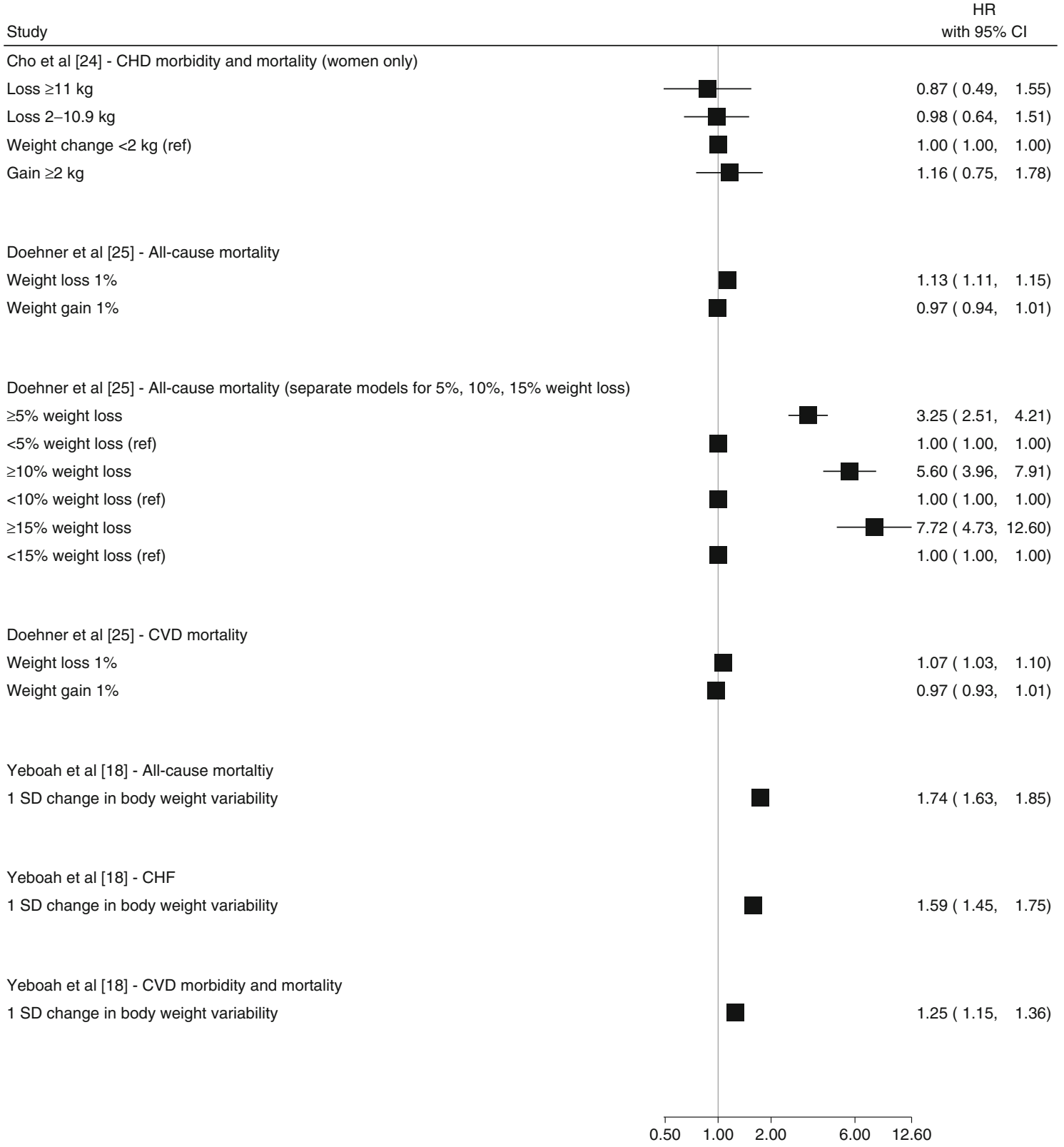

Fig. 5 Forest plot of HRs and 95\% CIs for the indicated outcome from studies of weight change among individuals with high CVD risk or other subgroups. Ref, referent group

all-cause mortality [19, 27]. However, studies showed mixed results regarding the associations between weight loss and incidence of CVD events and all-cause mortality. The only studies showing protective associations between weight loss and CVD were those concerning weight loss occurring over 1 year $[26,30]$. Studies of weight loss or weight variability over longer durations showed associations with increased risks of all-cause mortality [18, 19, 22, 27], although such risks were attenuated in some cases with the introduction of a 1-2 year lag in the risk period. Associations with mortality risks were more consistent in populations with elevated CVD risk $[18,25]$, and where weight loss was unintentional [17, 19]. Differences in the timing and extent of weight changes, the study populations with respect to CVD risk and diabetes progression, the method to mitigate confounding by unintentional weight loss, and the duration of follow-up contributed to heterogeneity in the results.

Weight loss intention influenced the effect of weight changes on health outcomes. However, while unintentional weight loss appeared to be associated with higher all-cause mortality risk, there was little evidence of protective effects of intentional weight loss. Weight loss intention was selfreported [17, 19, 28] and may not capture weight management behaviours, as details on changes in behaviours were not included in the studies. Participant-reported intention to lose weight does not rule out potential confounding by weight loss 
Fig. 6 Forest plot of HRs and $95 \%$ CIs for the indicated outcome from studies of intentional vs unintentional weight change. Ref, referent group

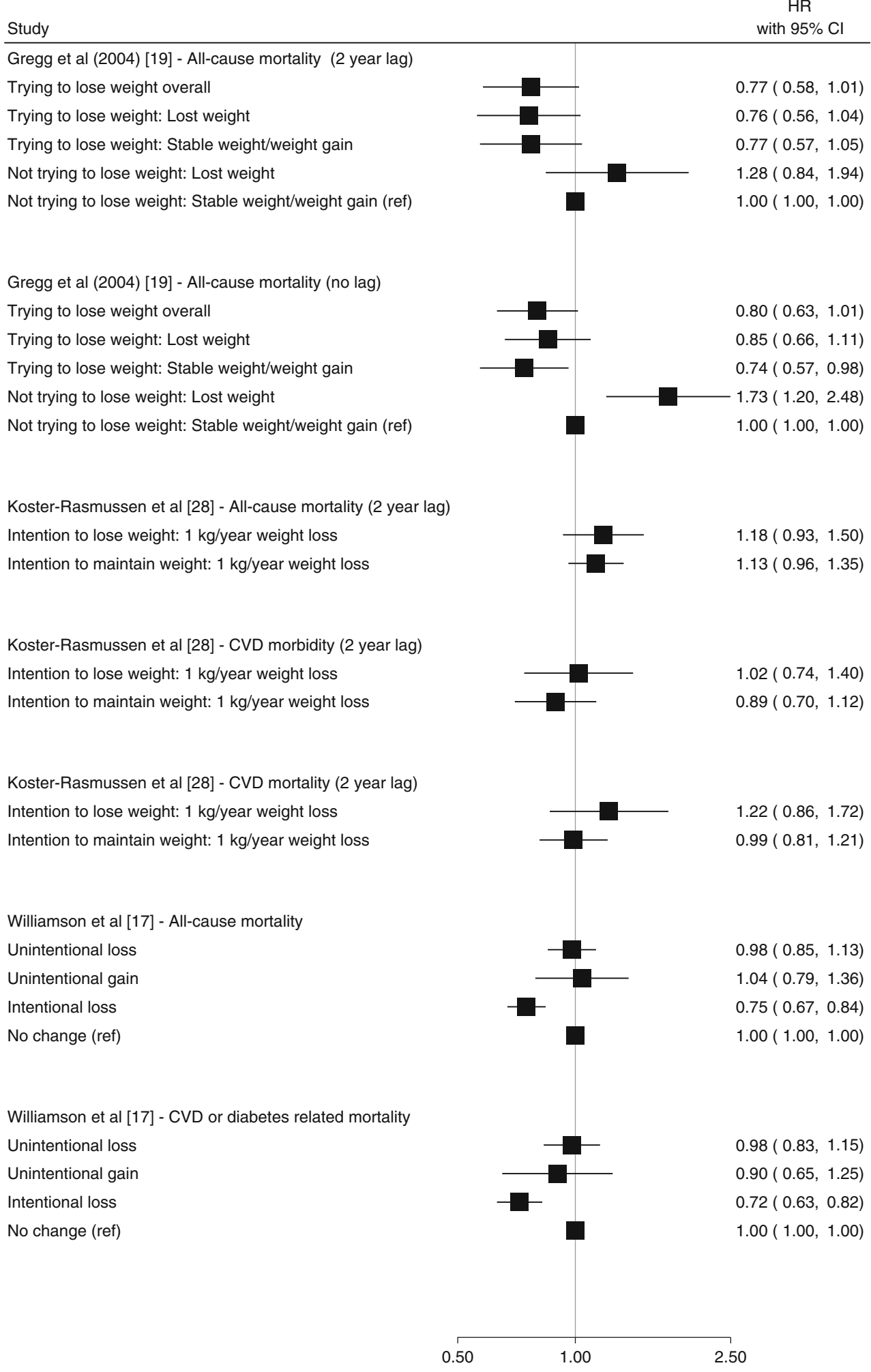

$0.77(0.58,1.01)$

$0.76(0.56,1.04)$

$0.77(0.57,1.05)$

$1.28(0.84,1.94)$

$00(1.00,1.00)$

$0.74(0.57,0.98)$

$1.73(1.20,2.48)$

$1.00(1.00,1.00)$

$1.02(0.74,1.40)$

$0.89(0.70,1.12)$

$1.04(0.79,1.36)$

$0.75(0.67,0.84)$

$1.00(1.00,1.00)$

$0.98(0.83,1.15)$

$0.90(0.65,1.25)$

$0.72(0.63,0.82)$

$1.00(1.00,1.00)$ caused by underlying disease. Furthermore, the high risk of bias identified in two of the studies that focused on weight loss intention [17, 19] limits interpretation. Other studies attempted to address confounding by unintentional weight loss by including a lag in the risk period for mortality.
Typically, the lag attenuated the results, suggesting that unintentional weight loss may otherwise inflate positive associations between weight loss and mortality.

Intervention studies are unlikely to be confounded by unintentional weight loss, although results were still inconsistent. 


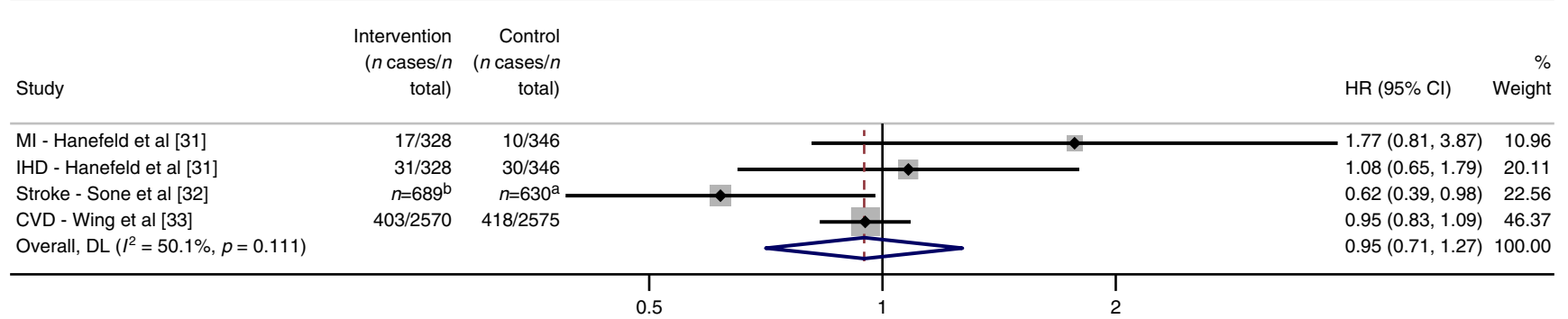

Fig. 7 Forest plot of meta-analysis of HRs and 95\% CIs from trials of behavioural interventions and incidence of CVD events, by study and outcome. IHD, ischaemic heart disease. Weight (\%) shows the relative percentage contribution of each study result to the meta-analysis. Vertical

One [33] of the three included trials reported significant weight loss among the intervention group, but nevertheless, the study reported no reduction in CVD events. As the interventions were similar, the heterogeneous findings may be related to differences in study populations, the achievement and maintenance of weight loss, and duration of follow-up. While Wing et al [33] reported no overall association between the behavioural intervention and incidence of CVD, post hoc stratified analyses showed that weight loss among the intervention group was associated with a lower hazard of CVD events [26]. Other post hoc research in this cohort identified heterogeneous intervention effects, depending on participants' glycaemic control and self-rated health [37, 38]. This heterogeneity may have contributed to the lack of an overall association seen in the original study results. Null findings may also be a product of low statistical power, and it is possible that longer duration of follow-up may be needed to identify the effects of interventions on CVD events in populations that are not specifically at high risk for CVD. For example, a reduction in CVD events was observed only after 23 years of follow-up in the Da Qing Diabetes Prevention Outcome Study [39], and in the Diabetes Prevention Program Outcomes Study, the investigators were not able to assess the effect of the intervention on CVD risk after 10 years of follow-up due to an insufficient number of events [40]. So, the 5-10 year follow-up of the included trials may not have been adequate to assess effects of behavioural interventions on CVD. Furthermore, the heterogeneity in the results between the studies should be considered when interpreting the meta-analysis. It is possible that other factors, including diabetes severity or participant characteristics, may influence the effect of weight-loss interventions on CVD and mortality outcomes but this has not been assessed. Risk of bias in the intervention studies was determined to be moderate in two and low in one (ESM Fig. 2), so bias is unlikely to be the primary reason for discrepant results between the trials. As we identified only three eligible studies that reported CVD events, this review highlights the need for more behavioural intervention studies with long-term follow-up.

In the observational studies, associations between weight change, CVD and mortality were influenced by baseline CVD dashed line indicates the meta-analysis point estimate. DL, DerSimonian and Laird method [16]. ${ }^{\mathrm{a}}$ Control group event rate: 9.52 events per 1000 person-years. ${ }^{\mathrm{b}}$ Intervention group event rate: 5.48 events per 1000 person-years

risk in the study population. Two studies using data from high-CVD-risk groups (ACCORD [18] and PROactive [25]) reported associations between weight loss, weight variability and higher risk of mortality. However, the ACCORD trial intervention included intensive use of glucose-lowering medication, and the observed associations may have been confounded by the effects of these medications on weight and CVD risk. While weight loss may have adverse effects among high-risk groups, it is also possible that the larger number of CVD events and higher premature mortality risk in these cohorts facilitated detection of associations between weight loss and events, which would be more difficult to detect in other cohorts. The results suggest that future behavioural intervention trials should consider testing effects of interventions on CVD separately among subgroups at higher CVD risk.

Studies of weight changes occurring over 1-2 years were more likely to show protective associations with CVD, while weight changes occurring over longer periods of time more often reported adverse associations [18, 22, 27]. This may be due to the fact that the majority of the studies with longer weight change duration did not include a lag time in the risk period for outcomes and may have been more susceptible to confounding by unintentional weight loss. Studies did not assess heterogeneity by differences in baseline BMI or by age, as has been done in other studies of the health effects of weight loss in the general population [41, 42]. Type 2 diabetes ascertainment was typically based on clinical diagnosis and did not include biochemical assessment of rare diabetes subtypes. Most studies did not exclude individuals with a history of cancer diagnosis, and it is possible that unintentional weight loss may be more common in this group.

We were unable to perform a meta-analysis of the observational studies due to heterogeneity in multiple study characteristics. Differences in study populations, outcomes, exposures and baseline CVD risk also complicated the narrative synthesis. As we only included published peer-reviewed research, this review is subject to publication bias if relevant unpublished works were excluded. We only included studies that included CVD 
events or mortality as an outcome; therefore, this review is not inclusive of behavioural intervention trials that focus on intermediate outcomes such as cardiovascular risk factors.

While there is compelling evidence that weight loss following bariatric surgery reduces risk of CVD [3], we have shown that the impact of non-surgical weight loss on CVD and mortality remains unclear. Weight gain was consistently associated with higher risk of CVD events but findings for the impact of weight loss are conflicting. Trial evidence of the effect on CVD endpoints of behavioural interventions targeting weight loss was also inconsistent and it remains unclear which subgroups of patients benefit most.

Conclusions Preventing weight gain among people with type 2 diabetes may help to reduce long-term burdens of CVD and premature mortality. While evidence accumulates for the benefits of weight loss on risk factors in the short term, there is limited evidence that existing behavioural approaches to achieving weight loss deliver long-term cardiovascular health benefits. In order to target weight-loss interventions efficiently, research is needed to identify patient groups that will achieve lower CVD event rates following weight loss, and to determine how much weight loss should be achieved and for how long this should be maintained. This evidence will help to most effectively allocate resources to improve longterm outcomes for people with diabetes.

Supplementary Information The online version contains peer-reviewed but unedited supplementary material available at https://doi.org/10.1007/ s00125-021-05605-1.

Acknowledgements We acknowledge V. M. Phillips, medical librarian at the University of Cambridge School of Clinical Medicine, who consulted on the keywords and terms used in the database searches.

Data availability The data from this study are available from the corresponding author on reasonable request.

Funding This work received MRC Epidemiology Unit core funding MC_UU_12015/4 and NIHR PGfAR RP PG 0216 20010. The study sponsors had no role in the study design, analysis, interpretation of data or the writing of this report.

Authors' relationships and activities SG reports grants from the Wellcome Trust, MRC, NIHR, NIHR Health Technology Assessment Programme, NHS R\&D and the University of Aarhus (Denmark). Outside the submitted work he also reports receiving fees from Novo Nordisk, AstraZeneca and Napp for speaking at postgraduate education meetings, support to attend a scientific meeting from Napp, and an honorarium and reimbursement of travel expenses from Eli Lilly associated with membership of an independent data monitoring committee for a randomised trial of a medication to lower glucose. ALA is principal investigator on two trials where research costs are funded by national research councils (NIHR and the National Prevention Research Initiative) and where the intervention is provided at no cost by Weight Watchers International. Other authors declare that there are no relationships or activities that might bias, or be perceived to bias, their work.
Contribution statement JS and EL wrote the study protocol, with SG and AA providing feedback. JS, EL, YW, AE and GN screened the articles for inclusion and performed data extraction. JS performed the data synthesis and meta-analysis, generated all data in the manuscript, wrote the manuscript and edited the manuscript. JS, SG, AA, EL, YW, AE and GN edited the manuscript and approved of the final version. JS is the guarantor of this work.

Open Access This article is licensed under a Creative Commons Attribution 4.0 International License, which permits use, sharing, adaptation, distribution and reproduction in any medium or format, as long as you give appropriate credit to the original author(s) and the source, provide a link to the Creative Commons licence, and indicate if changes were made. The images or other third party material in this article are included in the article's Creative Commons licence, unless indicated otherwise in a credit line to the material. If material is not included in the article's Creative Commons licence and your intended use is not permitted by statutory regulation or exceeds the permitted use, you will need to obtain permission directly from the copyright holder. To view a copy of this licence, visit http://creativecommons.org/licenses/by/4.0/.

\section{References}

1. Lean MEJ, Leslie WS, Barnes AC et al (2019) Durability of a primary care-led weight-management intervention for remission of type 2 diabetes: 2-year results of the DiRECT open-label, cluster-randomised trial. Lancet Diabetes Endocrinol 7(5):344355. https://doi.org/10.1016/S2213-8587(19)30068-3

2. Wing RR, Lang W, Wadden TA et al (2011) Benefits of modest weight loss in improving cardiovascular risk factors in overweight and obese individuals with type 2 diabetes. Diabetes Care 34(7): 1481-1486. https://doi.org/10.2337/dc10-2415

3. Eliasson B, Liakopoulos V, Franzén S et al (2015) Cardiovascular disease and mortality in patients with type 2 diabetes after bariatric surgery in Sweden: a nationwide, matched, observational cohort study. Lancet Diabetes Endocrinol 3(11):847-854. https://doi.org/ 10.1016/S2213-8587(15)00334-4

4. Nauck MA, Meier JJ, Cavender MA, Abd El Aziz M, Drucker DJ (2017) Cardiovascular actions and clinical outcomes with glucagon-like Peptide-1 receptor agonists and dipeptidyl Peptidase-4 inhibitors. Circulation 136(9):849-870. https://doi. org/10.1161/CIRCULATIONAHA.117.028136

5. Zinman B, Wanner C, Lachin JM et al (2015) Empagliflozin, cardiovascular outcomes, and mortality in type 2 diabetes. N Engl J Med 373(22):2117-2128. https://doi.org/10.1056/ NEJMoa 1504720

6. Moher D, Liberati A, Tetzlaff J, Altman DG (2009) Preferred reporting items for systematic reviews and meta-analyses: the PRISMA statement. PLoS Med 6(7):e1000097. https://doi.org/10. 1371/journal.pmed.1000097

7. Sweeney TE, Morton JM (2013) The human gut microbiome: a review of the effect of obesity and surgically induced weight loss. JAMA Surg 148(6):563-569. https://doi.org/10.1001/jamasurg.2013.5

8. Luijten J, Vugts G, Nieuwenhuijzen GAP, Luyer MDP (2019) The importance of the microbiome in bariatric surgery: a systematic review. Obes Surg 29(7):2338-2349. https://doi.org/10.1007/ s11695-019-03863-y

9. van den Munckhof ICL, Kurilshikov A, Ter Horst R, Riksen NP, Joosten LAB, Zhernakova A et al (2018) Role of gut microbiota in chronic low-grade inflammation as potential driver for 
atherosclerotic cardiovascular disease: a systematic review of human studies. Obes Rev 19(12):1719-1734. https://doi.org/10. 1111/obr. 12750

10. SIGN. SIGN Search Filters: Health Improvement Scotland; Available from: https://www.sign.ac.uk/what-we-do/ methodology/search-filters/. Accessed 28 Oct 2020

11. VHI Ltd. Covidence 2020. Available from: https://www.covidence. org/ Accessed 28 Oct 2020

12. The Cochrane Public Health Group. Cochrane Public Health Group Data Extraction and Assessment Template. 2011. https://ph. cochrane.org/sites/ph.cochrane.org/files/public/uploads/ CPHGDataextractiontemplate_0.docx. Accessed 1 Jun 2020

13. Schultz WM, Kelli HM, Lisko JC et al (2018) Socioeconomic status and cardiovascular outcomes: challenges and interventions. Circulation 137(20):2166-2178. https://doi.org/10.1161/ CIRCULATIONAHA.117.029652

14. Hoffmann TC, Glasziou PP, Boutron I et al (2014) Better reporting of interventions: template for intervention description and replication (TIDieR) checklist and guide. Bmj 348:g1687. https://doi.org/ 10.1136/bmj.g1687

15. McGuinness LA, Higgins JPT (2020) Risk-of-bias VISualization (robvis): An R package and Shiny web app for visualizing risk-ofbias assessments. Research synthesis methods. https://www. riskofbias.info/welcome/robvis-visualization-tool. Accessed 1 Oct 2020

16. DerSimonian R, Laird N (1986) Meta-analysis in clinical trials. Control Clin Trials 7(3):177-188

17. Williamson DF, Thompson TJ, Thun M, Flanders D, Pamuk E, Byers T (2000) Intentional weight loss and mortality among overweight individuals with diabetes. Diabetes Care 23(10):1499-1504. https://doi.org/10.2337/diacare.23.10.1499

18. Yeboah P, Hsu FC, Bertoni AG, Yeboah J (2019) Body mass index, change in weight, body weight variability and outcomes in type 2 diabetes mellitus (from the ACCORD trial). Am J Cardiol 123(4): 576-581. https://doi.org/10.1016/j.amjcard.2018.11.016

19. Gregg EW, Gerzoff RB, Thompson TJ, Williamson DF (2004) Trying to lose weight, losing weight, and 9-year mortality in overweight U.S. adults with diabetes. Diabetes Care 27(3):657-662. https://doi.org/10.2337/diacare.27.3.657

20. Hanson RL, Jacobsson LT, McCance DR et al (1996) Weight fluctuation, mortality and vascular disease in Pima Indians. Int J Obes Relat Metab Disord 20(5):463-471

21. Aucott LS, Philip S, Avenell A, Afolabi E, Sattar N, Wild S (2016) Patterns of weight change after the diagnosis of type 2 diabetes in Scotland and their relationship with glycaemic control, mortality and cardiovascular outcomes: a retrospective cohort study. BMJ Open 6(7):e010836. https://doi.org/10.1136/bmjopen-2015-010836

22. Bangalore S, Fayyad R, DeMicco DA, Colhoun HM, Waters DD (2018) Body weight variability and cardiovascular outcomes in patients with type 2 diabetes mellitus. Circ Cardiovasc Qual Outcomes 11(11):e004724. https://doi.org/10.1161/ CIRCOUTCOMES.118.004724

23. Bodegard J, Sundstrom J, Svennblad B, Ostgren CJ, Nilsson PM, Johansson G (2013) Changes in body mass index following newly diagnosed type 2 diabetes and risk of cardiovascular mortality: a cohort study of 8486 primary-care patients. Diabetes Metab 39(4): 306-313. https://doi.org/10.1016/j.diabet.2013.05.004

24. Cho E, Manson JE, Stampfer MJ et al (2002) A prospective study of obesity and risk of coronary heart disease among diabetic women. Diabetes Care 25(7):1142-1148. https://doi.org/10.2337/diacare. 25.7.1142

25. Doehner W, Erdmann E, Cairns R et al (2012) Inverse relation of body weight and weight change with mortality and morbidity in patients with type 2 diabetes and cardiovascular co-morbidity: an analysis of the PROactive study population. Int J Cardiol 162(1): 20-26. https://doi.org/10.1016/j.ijcard.2011.09.039
26. Gregg EW, Jakicic JM, Blackburn G et al (2016) Association of the magnitude of weight loss and changes in physical fitness with longterm cardiovascular disease outcomes in overweight or obese people with type 2 diabetes: a post-hoc analysis of the look AHEAD randomised clinical trial. Lancet Diabetes Endocrinol 4(11):913-921

27. Kim MK, Han K, Koh ES et al (2019) Weight change and mortality and cardiovascular outcomes in patients with new-onset diabetes mellitus: a nationwide cohort study. Cardiovasc Diabetol 18(1):36

28. Koster-Rasmussen R, Simonsen MK, Siersma V, Henriksen JE, Heitmann BL, de Fine Olivarius N (2016) Intentional weight loss and longevity in overweight patients with type 2 diabetes: a population-based cohort study. PLoS One 11(1):e0146889. https://doi.org/10.1371/journal.pone.0146889

29. Nunes AP, Iglay K, Radican L et al (2017) Hypoglycaemia seriousness and weight gain as determinants of cardiovascular disease outcomes among sulfonylurea users. Diabetes Obes Metab 19(10): 1425-1435. https://doi.org/10.1111/dom. 13000

30. Strelitz J, Ahern AL, Long GH et al (2019) Moderate weight change following diabetes diagnosis and 10 year incidence of cardiovascular disease and mortality. Diabetologia 62(8):13911402. https://doi.org/10.1007/s00125-019-4886-1

31. Hanefeld M, Fischer S, Schmechel H et al (1991) Diabetes intervention study. Multi-intervention trial in newly diagnosed NIDDM. Diabetes Care 14(4):308-317. https://doi.org/10.2337/diacare.14.4.308

32. Sone $\mathrm{H}$, Tanaka $\mathrm{S}$, Iimuro $\mathrm{S}$ et al (2010) Long-term lifestyle intervention lowers the incidence of stroke in Japanese patients with type 2 diabetes: a nationwide multicentre randomised controlled trial (the Japan diabetes complications study). Diabetologia 53(3): 419-428. https://doi.org/10.1007/s00125-009-1622-2

33. Wing RR, Bolin P, Brancati FL et al (2013) Cardiovascular effects of intensive lifestyle intervention in type 2 diabetes. N Engl J Med 369(2):145-154. https://doi.org/10.1056/NEJMoa1212914

34. Colhoun HM, Betteridge DJ, Durrington PN et al (2004) Primary prevention of cardiovascular disease with atorvastatin in type 2 diabetes in the collaborative atorvastatin diabetes study (CARDS): multicentre randomised placebo-controlled trial. Lancet 364(9435): 685-696. https://doi.org/10.1016/S0140-6736(04)16895-5

35. Knopp RH, d'Emden M, Smilde JG, Pocock SJ (2006) Efficacy and safety of atorvastatin in the prevention of cardiovascular end points in subjects with type 2 diabetes: the atorvastatin study for prevention of coronary heart disease endpoints in non-insulin-dependent diabetes mellitus (ASPEN). Diabetes Care 29(7):1478-1485. https://doi.org/10.2337/dc05-2415

36. LaRosa JC, Grundy SM, Waters DD et al (2005) Intensive lipid lowering with atorvastatin in patients with stable coronary disease. N Engl J Med 352(14):1425-1435. https://doi.org/10.1056/ NEJMoa050461

37. Baum A, Scarpa J, Bruzelius E, Tamler R, Basu S, Faghmous J (2017) Targeting weight loss interventions to reduce cardiovascular complications of type 2 diabetes: a machine learning-based posthoc analysis of heterogeneous treatment effects in the look AHEAD trial. Lancet Diabetes Endocrinol 5(10):808-815. https://doi.org/ 10.1016/S2213-8587(17)30176-6

38. de Vries TI, Dorresteijn JAN, van der Graaf Y, Visseren FLJ, Westerink J (2019) Heterogeneity of treatment effects from an intensive lifestyle weight loss intervention on cardiovascular events in patients with type 2 diabetes: data from the look AHEAD trial. Diabetes Care 42(10):1988-1994. https://doi.org/10.2337/dc19-0776

39. Li G, Zhang P, Wang J et al (2014) Cardiovascular mortality, allcause mortality, and diabetes incidence after lifestyle intervention for people with impaired glucose tolerance in the Da Qing diabetes prevention study: a 23-year follow-up study. Lancet Diabetes Endocrinol 2(6):474-480. https://doi.org/10.1016/S2213-8587(14) 70057-9 
40. Orchard TJ, Temprosa M, Barrett-Connor E et al (2013) Long-term effects of the diabetes prevention program interventions on cardiovascular risk factors: a report from the DPP outcomes study. Diabet Med 30(1):46-55

41. Myrskylä M, Chang VW (2009) Weight change, initial BMI, and mortality among middle- and older-aged adults. Epidemiology 20(6):840-848. https://doi.org/10.1097/EDE.0b013e3181b5f520

42. Mulligan AA, Lentjes MAH, Luben RN, Wareham NJ, Khaw KT (2018) Weight change and 15 year mortality: results from the
European prospective investigation into Cancer in Norfolk (EPICNorfolk) cohort study. Eur J Epidemiol 33(1):37-53. https://doi. org/10.1007/s10654-017-0343-y

Publisher's note Springer Nature remains neutral with regard to jurisdictional claims in published maps and institutional affiliations. 